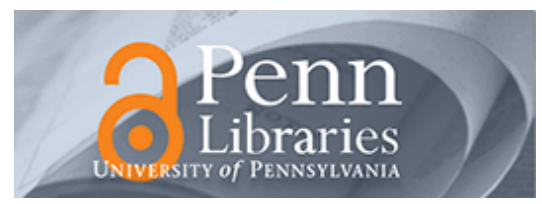

University of Pennsylvania ScholarlyCommons

$1-2013$

\title{
Variable Stiffness Legs for Robust, Efficient, and Stable Dynamic Running
}

\author{
Kevin C. Galloway \\ Harvard University \\ Jonathan E. Clark \\ Florida A\&M University / Florida State University \\ Daniel E. Koditschek \\ University of Pennsylvania, kod@seas.upenn.edu
}

Follow this and additional works at: https://repository.upenn.edu/ese_papers

Part of the Electrical and Computer Engineering Commons, and the Systems Engineering Commons

\section{Recommended Citation}

Kevin C. Galloway, Jonathan E. Clark, and Daniel E. Koditschek, "Variable Stiffness Legs for Robust, Efficient, and Stable Dynamic Running", . January 2013.

\section{BibTeX entry} @article\{Galloway-Journal_of_Mechanisms_and_Robots-2013, author $=\{$ Kevin C. Galloway and Jonathan E. Clark et al $\}$, title $=\{$ Variable Stiffness Legs for Robust, Efficient, and Stable Dynamic Running $\}$, booktitle $=\{$ Journal of Mechanisms and Robotics $\}$, year $=\{2013\}$, month $=\{$ January $\}$,

This work was partially supported by the NSF FIBR Grant \#0425878 and the IC Postdoctoral Fellow Program under Grant no. HM158204-1-2030.

This paper is posted at ScholarlyCommons. https://repository.upenn.edu/ese_papers/664

For more information, please contact repository@pobox.upenn.edu. 


\title{
Variable Stiffness Legs for Robust, Efficient, and Stable Dynamic Running
}

\author{
Abstract \\ Humans and animals adapt their leg impedance during running for both internal (e.g., loading) and \\ external (e.g., surface) changes. To date, the mechanical complexity of designing usefully robust tunable \\ passive compliance into legs has precluded their implementation on practical running robots. This work \\ describes the design of novel, structure-controlled stiffness legs for a hexapedal running robot to enable \\ runtime modification of leg stiffness in a small, lightweight, and rugged package. As part of this \\ investigation, we also study the effect of varying leg stiffness on the performance of a dynamical running \\ robot. \\ For more information: Kod*Lab \\ Disciplines \\ Electrical and Computer Engineering | Engineering | Systems Engineering \\ Comments \\ BibTeX entry \\ @article\{Galloway-Journal_of_Mechanisms_and_Robots-2013, author $=\{$ Kevin C. Galloway and Jonathan \\ E. Clark et al $\}$, title $=\{$ Variable Stiffness Legs for Robust, Efficient, and Stable Dynamic Running $\}$, booktitle \\ $=\{$ Journal of Mechanisms and Robotics $\}$, year $=\{2013\}$, month $=\{$ January $\}$, \\ This work was partially supported by the NSF FIBR Grant \#0425878 and the IC \\ Postdoctoral Fellow Program under Grant no. HM158204-1-2030.
}




\title{
Variable Stiffness Legs for Robust, Efficient, and Stable Dynamic Running
}

\author{
Kevin C. Galloway \\ Wyss Institute for \\ Biologically Inspired Engineering \\ Harvard University \\ Cambridge, MA 02138 \\ Email: kevin.galloway@wyss.harvard.edu
}

\author{
Jonathan E. Clark \\ Department of Mechanical Engineering \\ FAMU/FSU College of Engineering \\ Tallahassee, FL 32310 \\ Email: clarkj@eng.fsu.edu
}

\author{
Daniel E. Koditschek \\ GRASP Laboratory \\ Department of Electrical and Systems Engineering \\ University of Pennsylvania \\ Philadelphia, PA, 19104 \\ Email: kod@seas.upenn.edu
}

\begin{abstract}
Humans and animals adapt their leg impedance during running for both internal (e.g. loading) and external (e.g. surface) changes. To date the mechanical complexities of designing usefully robust tunable passive compliance into legs has precluded their implementation on practical running robots. This work describes the design of novel, structure-controlled stiffness legs for a hexapedal running robot to enable runtime modification of leg stiffness in a small, lightweight, and rugged package. As part of this investigation, we also study the effect of varying leg stiffness on the performance of a dynamical running robot.
\end{abstract}

\section{Introduction}

Running animals utilize their legs to run effectively over a large range of terrains. With each step the gravitational and kinetic energy of their bodies is transformed via their leg muscles, tendons and ligaments into strain energy that is stored during the deceleration in the first half of stance and is returned, aided by muscle contraction, during the second half of stance to re-accelerate the body [1]. These dynamics of running can be modeled using the Spring Loaded Inverted Pendulum (SLIP) model [2,3] and, despite its simplicity, it accurately captures the ground reaction forces and the motion of the center of mass for a wide scope of animals [4]. This model has, in turn, been followed by the development of a range of dynamically running robots, first Raibert's hoppers adopting the literal SLIP morphology [5], and subsequently, fully powered autonomous platforms - first, stiff [6] and compliant-legged versions of the quadruped Scout [7,8], then the hexapods RHex [9] and Sprawlita [10], which were shown to 'anchor' the SLIP dynamics ableit in a very different morphology. Despite the success of these robots, their performance, especially in variable terrains, pales in comparison to their biological inspirations.

The ability of animals to run over large obstacles and over a variety of terrains is aided by their ability to modulate the stiffness of their compliant legs in reaction to changes in their environment [11]. Apparently these animals can operate at or near optimal conditions for passive, dynamic self-stabilization because the viscoelastic properties of their passive mechanisms, termed preflexes [12], help stabilize their locomotion against perturbations [13], apparently with response times faster than could be attributed to reflex action [14]. We are interested in designing robotic limbs that can mimic or exceed the performance of animal legs by understanding the animal legs' functional properties and by relating their passive properties to the controller design and ultimately a correspondingly designed machine's dynamic performance.

Several robotic designers have attempted to imbue their platforms with mechanically adaptable impedance properties to provide both energetic efficiency and the flexibility to deal with changing conditions. Variable stiffness limbs have been developed for walking $[15,16]$ and more recently for a bipedal runner $[17,18]$. Although biological precedent and basic dynamic systems theory suggest that tuned resonant running should improve the performance of these systems $[17,19]$, no robot has yet demonstrated this advantage in terms of speed, efficiency or stability for an autonomous runner.

For our investigation into the role of tunable leg impedance, we use the EduBot platform [21] shown in Fig. 1. Similar to its predecessor, RHex [9], EduBot uses six compli- 


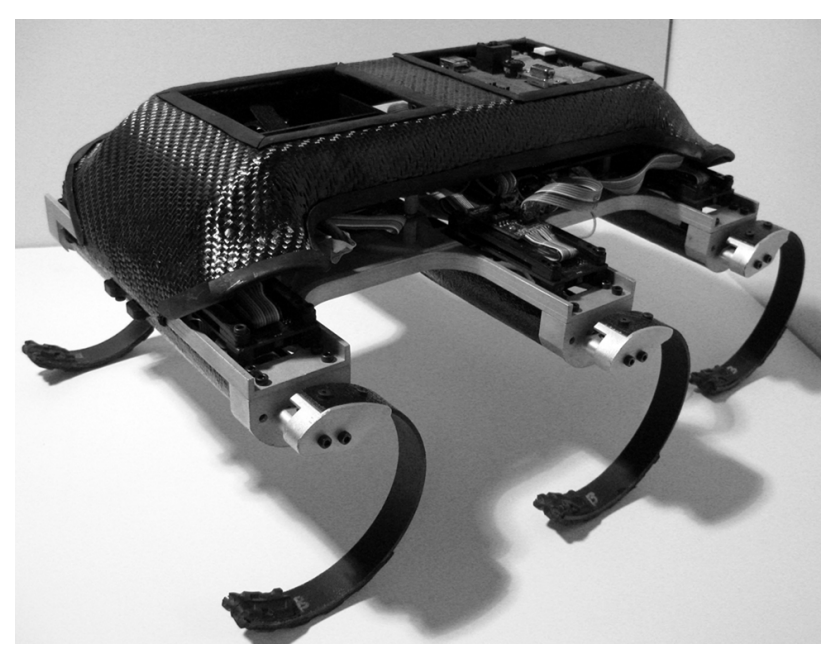

Fig. 1. EduBot [20], a hexapod robot considered for studying the effect of the legs with variable stiffness on the robot's dynamic stability

ant legs and a task-level open loop controller to run at several body lengths per second over rough terrain. Beyond the similarity to RHex, the EduBot platform was also selected for tunable leg integration because the mechanical configuration allows for direct integration of new leg designs without affecting sub-assemblies, and the robot size $(3 \mathrm{~kg})$ is easy to handle.

In this paper we describe the design and development of novel, structure-controlled variable stiffness legs for this robot. These legs overcome the size, weight, fragility, and efficiency problems encountered in earlier designs, enabling the autonomous operation of passive variable compliance legs on a running robot.

This paper is organized as follows. We provide in section II an overview of methods for implementing variable stiffness legs and describe the design of the new structurecontrolled variable stiffness leg and discuss its advantages over previous designs. In this section we also, present a Pseudo-Rigid Body (PRB) modeling approach for designing tunable legs to achieve desired stiffness ranges. Section III describes leg manufacturing methods and bench top experimental studies for characterizing leg stiffness range and resulting deflection path. In section IV, we present empirical evidence collected from EduBot running trials that captures the effect of leg stiffness on locomotion speed and specific resistance. Section V summarizes the results of the paper and describes future work.

\section{Variable Stiffness Leg Design}

Traditionally, stiffness control in robotic mechanisms has been implemented at the motor level by adjusting the gains at individual joints. For dynamic tasks with significant impacts of unpredictable timing, such as running, the inherent power limitations and bandwidth delays in motor control have led to the development and adoption of passively compliant legs. The introduction of series elastic actuators [22] rather than stiffness control of the actuated joints has the ad- vantages of zero-lag (infinite bandwidth) and the possibility of energy storage and return.

Inspired by Raibert's work on simple dynamic hopping robots [5], mechanical leg springs have been incorporated into a number of legged platforms including the Scout [7], [8], Tekken [23], Kolt [24], Whegs [25, 26], Sprawlita [27], and RHex [28].

Our experience with dynamic running legged robots suggests that properly designed and tuned passive elements in the legs benefit locomotion along at least three distinct behavioral axes:

Physical Robustness: Leg springs act as low-pass filters on the impact forces from ground contact, reducing the shock experience by the robot's body, significantly increasing the overall system's physical robustness.

Energetic Efficiency: Springs act in concert with the rhythmically excited actuators to act as a tuned harmonic system, increasing the efficiency of locomotion.

Dynamic Stability: Properly designed spring elements alter the dynamics of the overall mechanical plant, thereby contributing to the overall stability of the robot against perturbative forces.

Both the Sprawl robots and early version of RHex (e.g., compare [29] to subsequent vestibular [30] and visual [31] endowments) run in an extroceptively open loop manner. A simple feed-forward oscillatory 'clock' excites the legs, and it is the interaction of the passive mechanical system with the substrate that induces convergent forward locomotion. Even in the face of significant out-of-plane perturbations or rough terrain these robots run in a dynamically stable manner. Although the complexity of interaction of the dynamics and the distributed compliance in the legs has thus far precluded a clear analytical understanding of how to tune the legs for optimal stability, our empirical experience has shown us that small changes in the magnitude or orientation of the spring elements has a dramatic effect on the stability of the resulting motion [32,33].

Research suggests that variable compliance can and should increase efficiency and robustness of runners [19], [17]. The RHex leg compliant C-shaped design [34] has proven very successful, but offers only one effective stiffness. The approach taken in this work is to extend the capabilities of an already proven dynamic legged locomotion system by adding variable compliant legs capable of offering a factor of two change in stiffness to improve gait control and efficiency over a range of forward speeds and differing terrains. In the process, we seek to better understand the role of variable leg stiffness in stabilizing and propelling dynamic legged robots.

\subsection{Variable Passive Compliant Actuators}

Variable passive compliant actuators come in several configurations including: an antagonistic set-up of 2-nonlinear springs, mechanical stiffness control, and structurecontrolled stiffness [35]. 
Recently Van Ham et al. [15] and Hurst et al. [17] have developed tunable stiffness joints that feature an antagonistic set-up of two non-linear passive actuators. The PPAM (Pleated Pneumatic Artificial Muscles) [15] is a muscle-like actuator that uses a pair of opposing pleated membranes that contract longitudinally when pressurized with air. The actuation technology offers a very favorable power to weight ratio; however, the high cost of pressurized air makes it an unlikely candidate for autonomous locomotion. The Biped with Mechanically Adjustable Series Compliance (BiMASC) is the first leg that we are aware of that was designed with the intent of being a variable mechanical stiffness leg for a dynamic running robot $[17,18]$. The design uses an antagonistic spring arrangement of non-linear fiberglass springs and a complex system of pulleys and cables to adjust joint stiffness. Its final configuration weighed approximately 30 $\mathrm{kg}(66 \mathrm{lbs})$ and stood about 1 meter tall. This prototype revealed that such complex methods of adjusting leg stiffness through antagonistic springs will not likely offer an efficient, much less robust means of energy storage. Its creator found significant energy losses as joint deflection only causes one spring to compress to store energy while the other relaxes to transfer energy into the compressing spring. Furthermore, the antagonistic spring arrangement creates significant internal forces that increase the friction of the system and necessitate stronger (i.e. heavier) parts to support these loads.

Another approach to creating a variable stiffness joint is the MACCEPA (Mechanically Adjustable Compliance and Controllable Equilibrium Position Actuator) [36], in which joint stiffness is controlled by two servo motors; one adjusts the angle of a lever arm which sets the equilibrium point and the other pretensions the spring independent of the equilibrium position. The MACCEPA is a simple design that works well for the controlled passive walking for which it was designed; however, the energetic and weight cost of supporting two motors to control a single joint stiffness makes it difficult to implement on a dynamic running robot with small, light legs.

The third common type of compliant actuation, known as structure-controlled stiffness, is a variable compliant method that changes the active structure of an elastic element such as a helical spring or a bending beam. Usually compliance is changed by adjusting the active length of a spring or the deflection point on a beam. Several groups have developed structure-controlled stiffness mechanisms [37-39], though none of them have been applied toward the development of autonomous dynamic legged locomotion systems.

In this work, our goal is to design a structure-controlled stiffness leg with properly tuned passive-mechanical properties in three dimensions, that can adapt these properties for efficient running at a number of forward speeds, loads, or surface conditions. We selected this stiffness control method as it can yield simple, robust mechanisms that offer 1) large stiffness ranges, 2) scalability, 3) constant passive compliant behavior (i.e., once the tuning mechanism is locked into position), and 4) light weight solutions since only a single tuning actuator is typically needed.
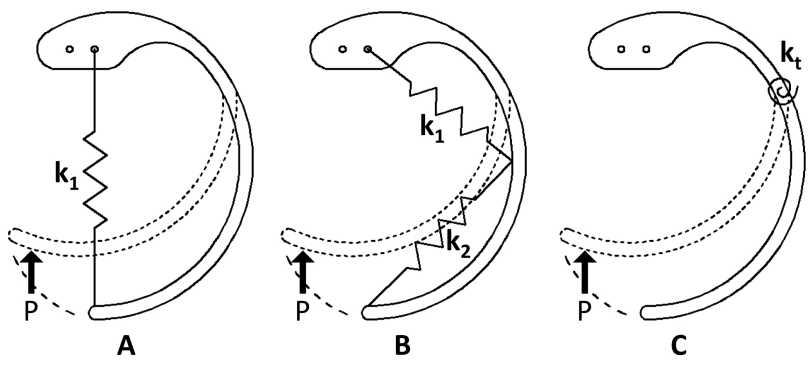

Fig. 2. Illustrations of the different spring models used to understand C-leg compliance under load a load, P. (A) Linear model (B) 2-orthogonal spring model $(C)$ Pseduo-rigid-body model where stiffness is characterized by a single torsional spring.

\subsection{Structure-Controlled Stiffness Leg Model}

As a base point for our variable-stiffness leg design we chose the current passive limbs of the RHex-style robot, EduBot, which consists of a rigid body and 6 compliant legs that each have one independently actuated revolute degree of freedom [28]. There have been several iterations on the compliant leg design for RHex [40]. The initial legs were built from a curved rod of delrin that was quickly abandoned for its low compliance and its fragility. The second major iteration was a 4-bar linkage design where the compliance was generated by the deformation of two fiberglass links of the mechanism [41]. This planar mechanism was easier to model, and had better deflection properties, but still had robustness issues. The current leg design is a semi-circular shaped fiberglass beam. The curved shape of the leg aids in standing from rest, and allows for the contact point to roll a small amount during stance.

Despite its success, little work has been conducted to understand C-leg's non-linear behavior under load. For the purposes of modeling, it has typically been simplified to a single linear spring even though under load, the leg end clearly deflects in 2 dimensions (see Fig. 2A). In 2005, Lin modeled this as a 2 DOF system by two orthogonally placed linear springs (see Fig. 2B) [42]. Although, the two spring approach captures the force-deflection behavior of the compliant leg, this is a difficult model to work with due to the number of parameters needed to specify the orientation and magnitude of the springs. We propose a new model to capture the spatial compliant properties of the leg in the sagittal plane (see Fig. 2C) and the lateral direction using a combination of PRB model and standard beam bending theory.

\subsubsection{Compliance in the Sagittal Plane}

In the PRB model, flexible members are represented as rigid links connected via pin joints with torsional springs (see Fig. 3) [43]. This approach was chosen for two reasons. First, the path followed by the leg end, or toe, is nearly circular. Thus, representing the leg stiffness as a torsional spring best captures the large, curved deflections of the leg under load. Second, the PRB model offers design and time saving advantages. For example, it is significantly easier to estimate the leg stiffness for different configurations and dimensions 


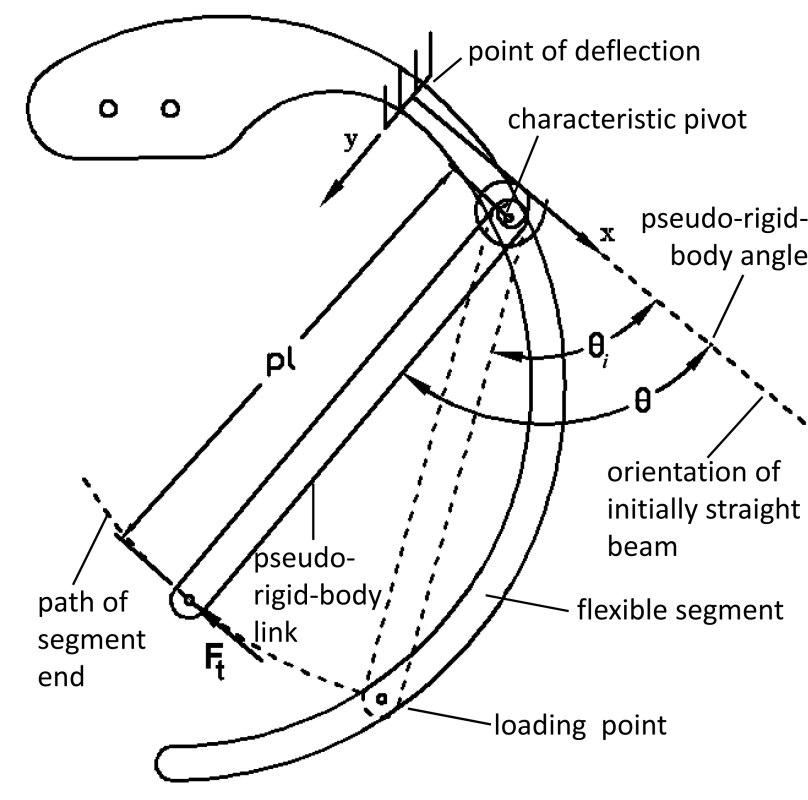

Fig. 3. Pseudo-rigid-body model applied to the C-leg. Adapted from [43].

using the PRB model than it is to update a solid model and constraints in a finite element program.

In this model the initial curvature and the length of the leg link are related through the non-dimensionalized parameter

$$
k_{o}=\frac{l}{R_{i}}
$$

where $l$ is the length measured along the centroidal axis of the leg from the point of deflection to the loading point, and $R_{i}$ is the initial curvature of the curved beam. Fig. 3 details the components of the PRB model where the characteristic radius factor, $\rho$, is used to determine the location of the the characteristic pivot and the length of the pseudo-rigid-body link. The PRB angle, $\Theta$, specifies the angle of the PRB link while, $\Theta_{i}$, defines the initial angle of the PRB link. Detailed explanations of the PRB model can be found in [43]; however, for the purposes of this paper we are primarily interested in the torsional spring constant. $K_{t}$ which is given by

$$
K_{t}=\rho K_{\Theta} \frac{E I_{\text {sagittal }}}{l}
$$

where $K_{\Theta}$ is the stiffness coefficient, E is the Young's modulus, and $I_{\text {sagittal }}$ is the second moment of inertia in the sagittal plane. For initially straight beams $K_{\Theta}$ is a function of the angle at which the load is applied. For initially curved beams and $k_{o}$ values 1.0 and higher, $K_{\Theta}$ is relatively constant for tangential and compressive beam loading. This allows $K_{\Theta}$ to be approximated from $k_{o}$. In the same way, for given $k_{o}$

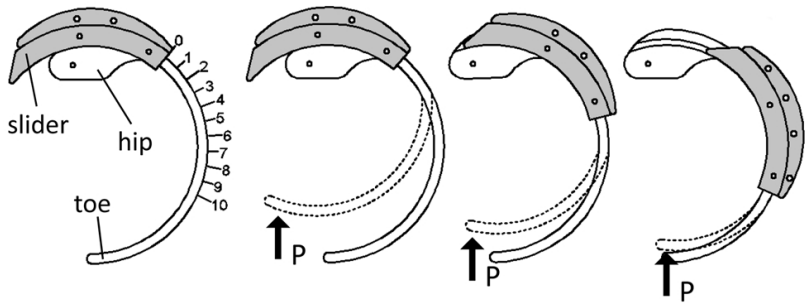

Fig. 4. An implementation of a structure-controlled stiffness mechanism applied to a C-leg.

values, $\rho$ can also be averaged for a range of loading conditions. These approximations have been captured in a simple look-up table in [44]. Therefore, E, I, and $k_{o}$ value are all that is needed to approximate the torsional spring constant in the PRB model.

\subsubsection{Compliance in the Lateral Direction}

The C-leg also has compliance in the lateral direction or the direction normal to the sagital plane. The leg stiffness in this direction, $K_{l}$ can be characterized by the standard cantilever beam bending equation

$$
K_{\text {lateral }}=3 \frac{E I_{\text {lateral }}}{L^{3}}
$$

where $\mathrm{L}$ is the linear distance from the point of deflection to the loading point, and $I_{\text {lateral }}$ is the moment of inertia in the lateral direction.

It is important to note that $K_{\text {sagittal }}$ and $K_{\text {lateral }}$ can be independently specified by changing the second moment of inertia. This feature increases design flexibility and allows one to adjust stiffness in the lateral direction independent of the sagittal plan. Our model assumes that small deflections in the lateral direction causes a negligible deflection in the sagital plane, allowing us to consider the motions effectively decoupled.

\subsubsection{Structure-Controlled Stiffness C-leg with a Rigid Slider}

To vary the leg stiffness of the C-leg, a robust sliding mechanism, labeled as 'slider' in Fig. 4, has been added. It is assumed that any portion of the leg that is covered by the slider is rigid, and the remaining exposed portion of the leg is compliant. In the sagittal plane, moving the slider changes the length of the PRB link and shifts the location and magnitude of the torsional spring constant. The same result is true for stiffness in the lateral direction where the slider changes the value of $L$ in equation 3 . In Fig. 4 , the slider can move continuously between the 10 evenly spaced markings (a $75^{\circ}$ sweep angle or an increment of $7.5^{\circ}$ per marking) where 0 is the most compliant configuration and 10 is the stiffest. Using the PRB model and the lateral stiffness equation, we can predictably design the tunable leg to operate within a range 


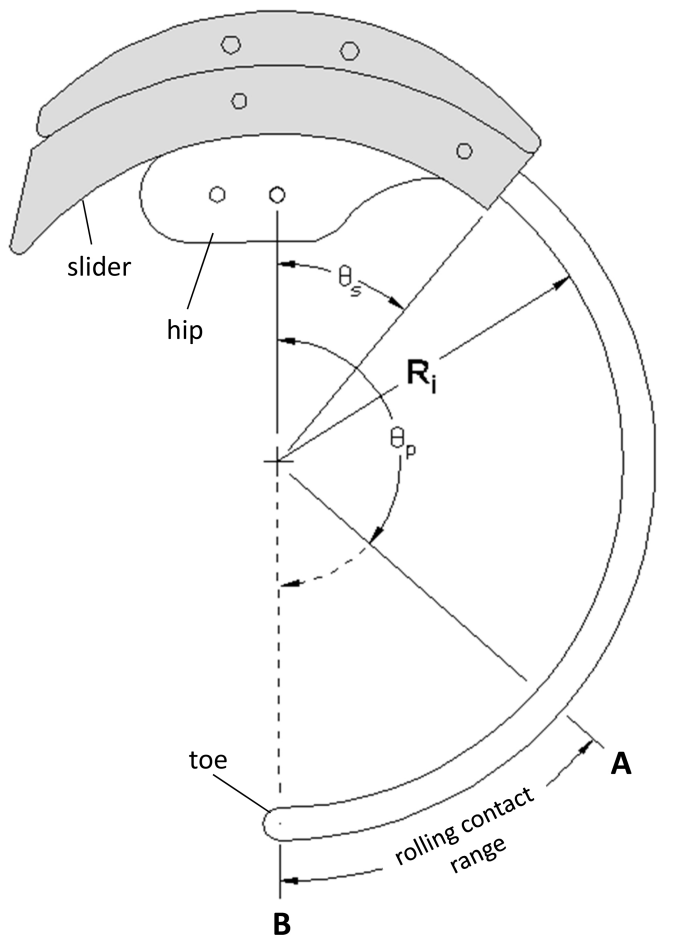

Fig. 5. Application of PRB-model to tunable leg where leg stiffness can be defined by the slider position and the loading point.

of stiffness's as long as a portion of the slider is supported by the hip region. For example, if the slider moves past the 10th marker it loses support from the hip region and the leg will begin to deflect from both ends of the slider.

\subsubsection{PRB Based Leg Model}

Thus far the PRB model has been presented with a single loading force where the loading point does not change. During operation, however, the loading point does in fact change. Generally the leg touches down at around point A (see Fig. 5) and rolls through to about point B during the loading and unloading phase. According to equation 2, the value of $K_{t}$ decreases from A to B because the value of $l$ increases. Although stiffness varies along the length of the leg, it is not really important to determine the exact stiffness of the leg for each loading point. During operation, the robot will be optimized for different stiffness settings. In the design stage, it is more important to consider the range of stiffness's, or relative stiffness of the leg. To calculate the range of stiffnesses for the C-leg presented in Fig. $5, k_{o}$ can also be represented as

$$
k_{o}=\theta_{s}-\theta_{p}
$$

where $\theta_{s}$ specifies the angular position of the slider or point of deflection, and $\theta_{p}$ species the loading point. Thus to design a C-leg that can vary it's stiffness in the sagittal plane by a factor or two there are several design variables in the model
Table 1. Material Properties

\begin{tabular}{cccc}
\hline Epoxie & $\mathrm{E}(M P a)$ & $S_{y}(M P a)$ & $\frac{S_{y}}{E} \times 1000$ \\
\hline TP-4000 & 690 & 21 & 30 \\
TP-4004 & 793 & 34 & 42.5 \\
TP-4007 & 2240 & 104 & 46 \\
\hline
\end{tabular}

that can be tuned including the range of $\theta_{s}$, the Young's modulus of the material, the moment of inertia $I_{\text {sagittal }}$, and the initial radius, $R_{i}$.

\section{Leg Manufacturing and Testing}

For the initial manufacture of the variable stiffness Cleg, it was important to select a method that would allow considerable design flexibility to test various materials and shapes quickly and economically, and one that offers the option of integrating parts to save volume and weight. These criteria were satisfied through Shape Deposition Manufacturing (SDM), a solid freeform fabrication process that systemically combines material deposition with material removal processes. The general SDM design principles and techniques are covered in detail in [45], and have been applied to several robotic systems [46-48]. SDM offers several advantages over traditional prototyping methods. Some of these include the ease of embedding components (i.e. actuators and electronics), the flexibility of combining dissimilar materials to create complex and robust compliant mechanisms, creating whole parts in a layered fashion, and eliminating custom tooling [48]. For the fabrication of the tunable leg designs, the SDM process offers the advantage of adjusting the leg shape and design variables noted earlier. The overall leg stiffness can be adjusted by choosing an epoxy from a family of materials (see Table 1) of different Young's moduli, $\mathrm{E}$, by changing the moment of inertia, I, or by changing the length of the slider. For example, the first tunable legs used for testing were fabricated from TP-4004 (Innovative Polymers, St. Johns, Michigan, USA) which offers a relatively low Young's modulus with a favorable strength-to-modulus ratio.

\subsection{Measuring Leg Stiffness \\ 3.1.1 Method}

The $K_{t}$ for the shape deposition manufactured C-leg was collected at each of the even numbered slider positions shown in Fig. 6. The leg was mounted to a Micos linear stage for ease of repeatability and the deflection of the leg against an AMTI HE6x6 force plate was visually captured. The linear stage has a resolution of one micrometer and is capable of traveling $80 \mathrm{~mm}$ at rates as high as $14 \mathrm{~mm} / \mathrm{s}$. The AMTI HE6x6 is a six axis force plate capable of measuring loads as large as $70 \mathrm{~N}$ at $200 \mathrm{hz}$ with 12 -bit resolution. Five measurements of the linear stage pressing the leg into the force plate at $10 \mathrm{~mm} / \mathrm{s}$ were collected.

The $K_{t}$ was obtained by marking evenly spaced colored 

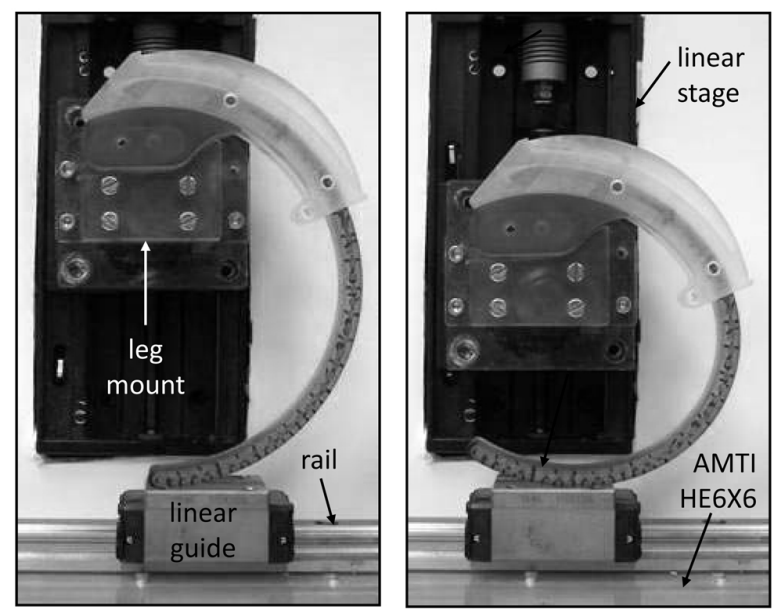

Fig. 6. Relaxed and compressed images of a C-leg in the experimental set-up

dots along the centroidal axis of the leg. An image capture system was created to compare the relaxed and compressed images to determine the loading point, point of deflection, the characteristic pivot, the arc length, $l$, and the value of the PRB-angle $\Theta-\Theta_{i}$.

The analytical $K_{t}$ was calculated by inputting the specified material properties, and $l$ into (2). The value for $K_{\Theta}$ and $\rho$ were determined from the look-up table in [44].

The experimental $K_{t}$ was calculated by first measuring the resultant torque, $T_{R}$, about the characterstic pivot using the force data and the horizontal and vertical distances measured from the characteristic pivot to the loading point. The resultant torque along with the the PRB-angle, $\Theta-\Theta_{i}$, were then applied to the torsional spring equation, (5), to determine the experimental torsional spring constant.

$$
K_{t}=\frac{T_{R}}{\Theta-\Theta_{i}}
$$

The stiffness in the lateral direction was determined by using the same force plate and linear stage. The toe was deflected in the lateral direction by pushing it into an obstruction rigidly anchored to the force plate. This experiment was repeated ten times for each even numbered slider position. A force-deflection graph was generated with the data, and a linear curve fit was applied to each experiment for a given slider position. The slopes of the linear curves were averaged to determine the average lateral leg stiffness for each slider position.

\subsubsection{Results}

For the sagittal plane stiffness, we found that the PRB model captured the behaviors of the leg under load reasonably well for a range of slider positions (see Fig. 7). The error between the analytical and average experimental torsional stiffness measurements was less then $3 \%$. For slider positions $0-8$, the analytical results fall within the error bars,

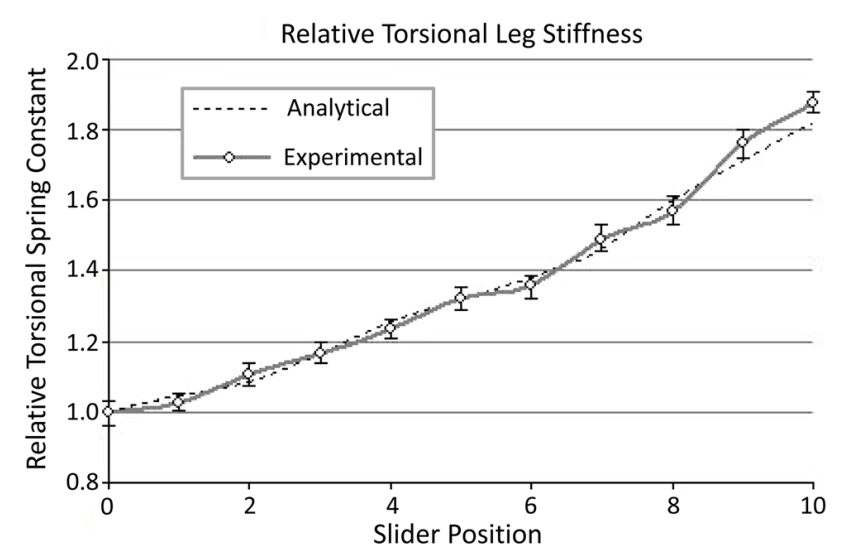

Fig. 7. Experimental validation of the PRB model for estimating torsional spring constant.

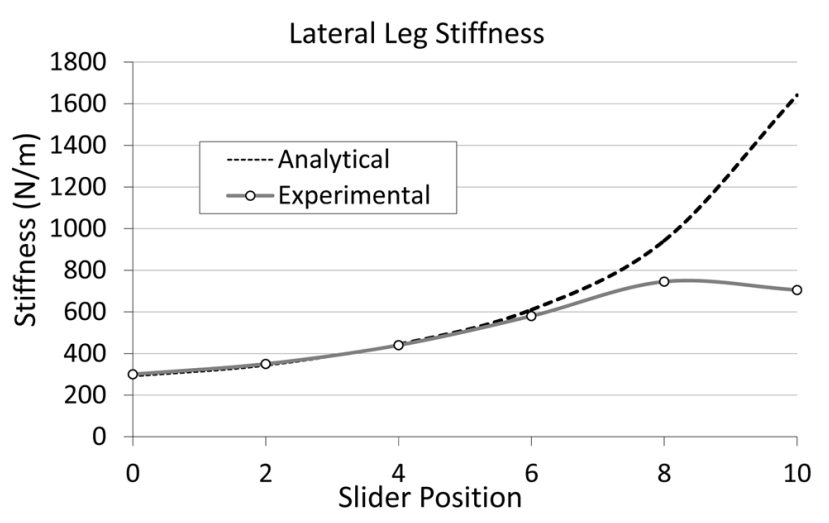

Fig. 8. Experimental validation of the cantilever beam bending model for estimating lateral leg stiffness

however this not the case for positions 9 and 10. This deviation can be attributed to deflection at the hip end of the slider. As mentioned earlier, as the slider moves to higher settings it is supported less and less by the hip region. For example, at slider position 10 , there are noticeable deflections at both ends of the slider. Since this behavoir is not accounted for in the PRB model, it introduces another source of error.

Deflection at the hip end of the slider is even more apparent and occurs earlier in the lateral stiffness experimental results. It is clear that the deviation between the analytical and experimental results begins near slider position 6 . As the slider moves to higher settings, deflections occur from both ends of the slider and the cantilever beam model is no longer valid.

Structure-controlled stiffness of the C-leg has its limitations, both in terms of directional coupling, and in the accuracy of the assumed linear model. While the PRB model estimation of torsional spring constant was close to the experimental results $(185 \%$ vs. $190 \%$ increase at the stiffest setting), in the lateral direction, the stiffness was considerably lower than predicted.

While the initial design demonstrated that the overall stiffness could be varied by as much as $190 \%$ there were at 
least three undesirable features coupled in the design 1) an altered tip deflection trajectory, 2) an increased probability of inelastic collisions, and 3) early fatigue failure of the legs.

Maintaining consistent tip trajectory for the continuous range of stiffness settings is an important feature to consider in a tunable leg. In our previous design each stiffness setting altered the deformation tendency of the leg spring. In other words, the deflection path of the leg spring would respond differently to applied loads depending on the stiffness setting. Such configurations make it difficult to determine whether a tunable leg performed better or worse due to the change in stiffness or to the altered deformation behavior.

One of the novel features of the original passive compliant C-shaped legs [40] is that they enable the robot to navigate rough terrain by allowing compliant ground contact anywhere along the length of the leg. A leg design with a rigid slider effectively limits the leg length that is capable absorbing impacts. This is important to consider as legs are generally stiffer at higher speeds where the potential for damage from collisions is greatest.

In our initial design, SDM was used to manufacture epoxy legs springs; however, we found that the fatigue life and energy density of epoxy is generally not optimal for the application of dynamic locomotion where legs are cyclically loaded under various and often unpredictable conditions. This was revealed when legs failed during early locomotion studies.

\subsection{Structure-Controlled Stiffness Leg with a Compli- ant Slider}

In this section we present a variable stiffness leg design that overcomes the drawbacks of the rigid slider configuration, improves the robustness, spring energy density, and incorporates an actuation system to enable autonomous stiffness adjustment.

\subsubsection{Compliant Slider Mechanism}

By replacing the bulky rigid slider with a light-weight flexible slider the mechanism not only saves weight, but also alters the manner in which the stiffness of the leg is changed. Rather than shortening the effective length of the compliant section with a rigid slider, the use of compliant slider in parallel to the leg can be though of a way of altering the effective moment of inertia of the affected portion of the leg. This in turn, reduces the change in location of the characteristic pivot, and the shape of the deflection path of the tip of the leg.

In the improved design shown in Figs. 9 and 10, the leg is anchored to an aluminum hip structure which also supports the drive mechanism. A thin, flexible rack is anchored to the back of the compliant slider to control its position without significantly altering the slider's stiffness. The position of the slider can be adjusted by activating a small, geared DC motor mounted to the hip, which simultaneously drives a nylon worm and spur gear (see Fig. 11). A small plastic guide is attached at one end of the spine and wraps around the Cleg. The guide holds the spine against the $\mathrm{C}-\mathrm{leg}$, and acts as

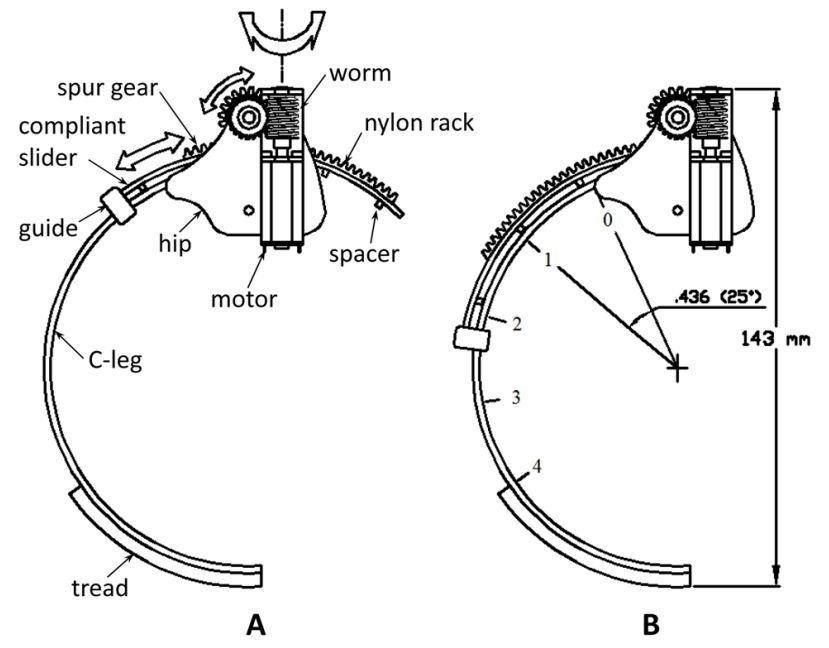

Fig. 9. Proposed new design: side view of tunable stiffness composite leg design. A) Illustrates the rotation directions of gears. B) Illustrates the spine adjusted to a higher stiffness setting.

a mechanical stop when the spine is actuated to the two extreme stiffness settings. The spacing between the C-leg and the compliant slider is approximately $1.7 \mathrm{~mm}$. It is important to maintain this spacing so that the two compliant elements deform together under load. To enforce this condition, small spacers were added to the inside surface of the compliant slider.

During operation, the motor can rotate clockwise or counterclockwise to move the slider through the continuous spectrum of leg stiffnesses. When the slider reaches a target stiffness setting, the motor shuts off, and the worm provides sufficient resistance to rotation in either direction; thus acting as a natural self-locking mechanism. Hence no power is required to maintain a desired leg stiffness during locomotion. This also results in a robust and efficient spring as there are no moving parts at a given stiffness setting. In its final configuration, the tunable C-leg has a $114 \mathrm{~mm}$ inner diameter and weighs less than 85 grams.

\subsubsection{Material Selection}

Based on our experience with epoxy based leg spring solutions we found it necessary to explore other materials and prototyping methods. With any passive compliant spring mechanism, the material property of the spring element and shape dictate its ability to store and return energy. Important material properties to consider for any elastic element include its density, Young's modulus, yield strength, fatigue life, energy storage density, and manufacturability.

We have prototyped legs with a variety of materials including plastic, nitinol, aluminum and glass fiber composites. These materials and some of their properties are listed in Table 2 where $\rho$ is the density, E is the Young's Modulus, $\mathrm{S}$ is the ultimate yield strength, $\mathrm{S} / \mathrm{E}$ is the yield strength to Young's modulus ratio, and $U$ is the specific strain energy of the material which is expressed as 


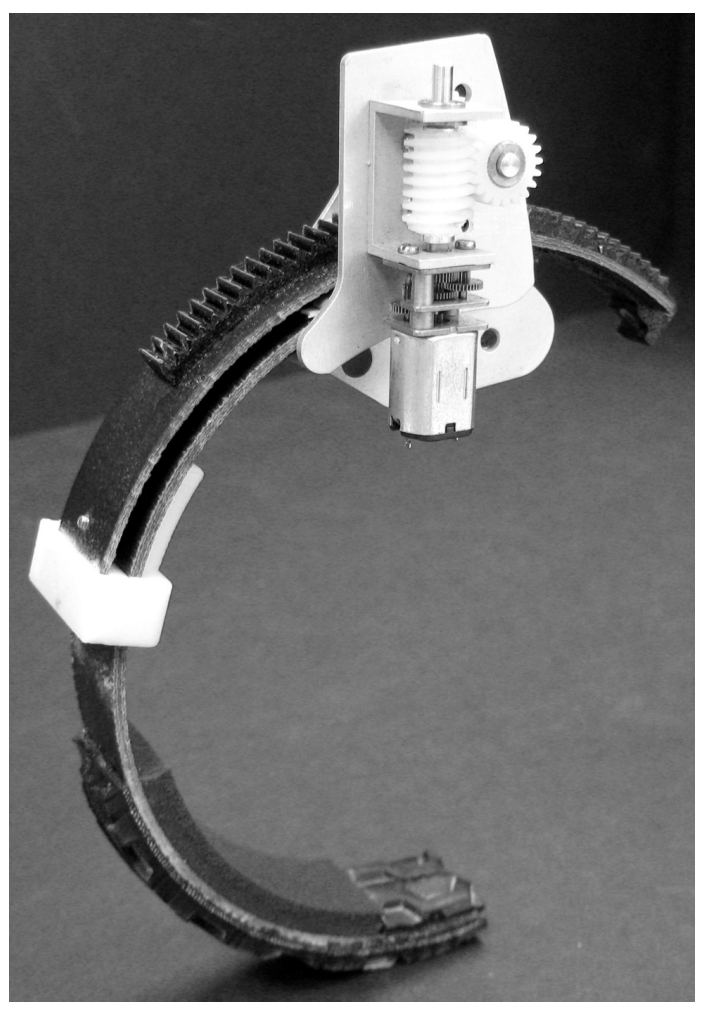

Fig. 10. Photograph of the prototyped variable stiffness C-leg.

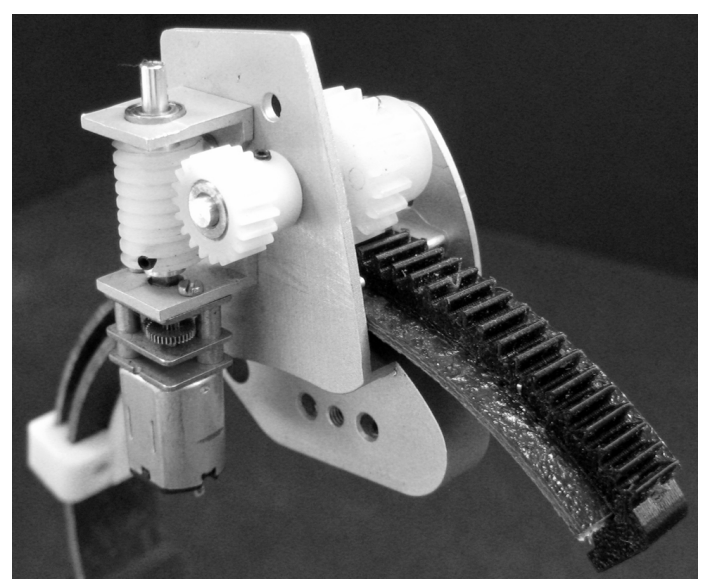

Fig. 11. Close-up of the active component.

\begin{tabular}{|l|c|c|c|c|c|c|}
\hline \multicolumn{1}{|c|}{ Material } & $\begin{array}{c}\rho \\
\text { (g/cc) } \\
\text { LIB }\end{array}$ & $\begin{array}{c}\text { E } \\
\text { (GPa) } \\
\text { LIB }\end{array}$ & $\begin{array}{c}\text { S } \\
\text { (Mpa) } \\
\text { HIB }\end{array}$ & $\begin{array}{c}\text { (S/E) } \\
\mathbf{x 1 0 ^ { 3 }} \\
\text { HIB }\end{array}$ & $\begin{array}{c}\mathbf{U} \\
\text { (kJ/kg) } \\
\text { HIB }\end{array}$ & $\begin{array}{c}\mathbf{S} \\
\text { (USD) } \\
\text { per leg } \\
\text { LIB }\end{array}$ \\
\hline Aluminum 7075-T6 & 2.81 & 71.7 & 500 & 7.0 & 1.24 & 1 \\
\hline Fiberglass (S2-6781) & 2.25 & 22 & $520+$ & 23.6 & 5.46 & 3 \\
\hline Nitinol & 6.50 & 1 & 1000 & 1333 & $6.8 *$ & $30+$ \\
\hline $\begin{array}{l}\text { Steel (4140 } \\
\text { Q\&T@400) }\end{array}$ & 7.75 & 207 & 238 & 1.1 & 0.04 & 2.5 \\
\hline TP-4004 (Epoxy) & 1.17 & 0.80 & 34 & 42.5 & 1.24 & 6 \\
\hline
\end{tabular}

*Duerig et al., "Engineering Aspects of Shape Memory Alloys," 1990.

Table 2. Comparison of material properties

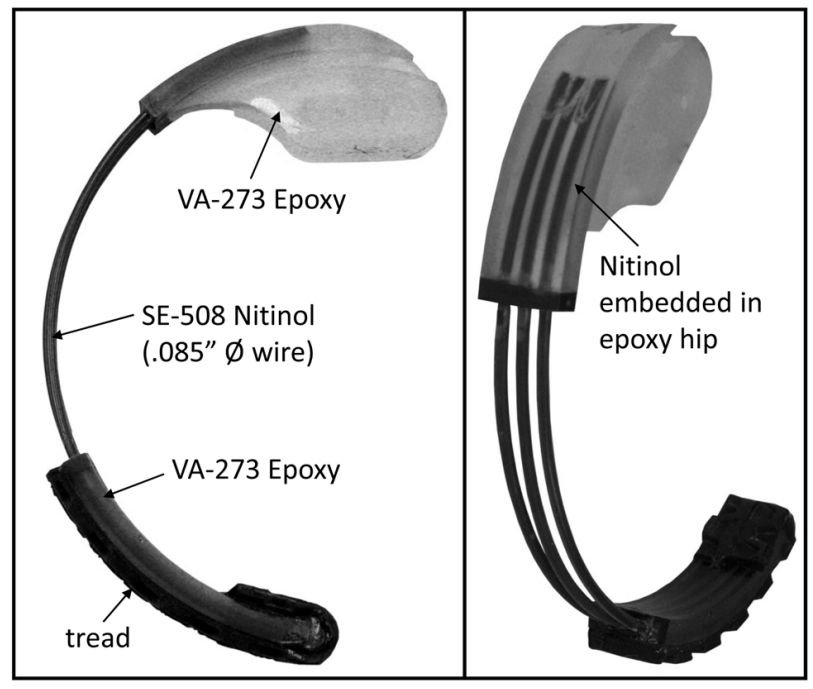

Fig. 12. Older design alternative: C-leg with a Nitinol spring element.

$$
U=\frac{S^{2}}{\rho E}
$$

It can be observed that the materials with the best specific strain energy capacity are those with a large yield strength and a low density and Young's modulus [49].

In our material studies, nitinol was considered for its high energy density and yield strength. As an elastic element, nitinol offers attractive properties including the ability to recover from bending strains as large as $10 \%$ without plasticly deforming (note: spring steel can manage about $0.2 \%$ strain before plastic deformation), and a low Young's modulus. However, nitinol has less desirable properties, including a high raw material cost, limited available stock geometries, hysteresis, and difficulty to form various geometries with tight tolerances. For exmaple, in order to achieve a desired curvature, nitinol must be clamped to a custom mold and baked at temperatures of $530^{\circ} \mathrm{C}$. Several legs were fabricated using this technique and SDM was used to embed them into a plastic hip structure (see Fig. 12); however, achieving consistant radius and stiffness values from leg to leg proved prohibitively difficult.

Composite laminate, specifically S2-6781 pre-preg fiberglass (Applied Vehicle Technologies, Inc., Indianapolis, IN), was eventually selected as the material of choice for several reasons including its relatively low density and Young's modulus, high yield strength, comparatively high specific strain energy capacity and low material cost. In addition to these properties, composite laminates expand the available design space by offering the ability to change the Young's modulus value. The isotropic nature of the other materials considered (i.e. metal and plastic) often leads to situations where a desired spring element geometry such as the moment of inertia, does not have the yield strength to withstand the 
demands of the intended environment which include stresses caused by changing payloads, speeds, irregular landings and collisions. Many composites, including the fiberglass composite chosen, are anisotropic and thus have properties that change depending on the orientation along which the property is measured [50]. By laying the plies in specific orientations during the manufacturing process, one can change the Young's modulus of a composite material by a factor of two or more. Thus, compared to isotropic materials, the stiffness of a spring element constructed from an anisotropic material is less dependent on the spring geometry.

\subsection{Leg Stiffness Model}

To estimate the stiffness range of the compliant C-leg with a compliant spine, we employ the PRB model as before. When the tunable leg is at the stiffest setting, we have found that the effective moment of inertia is best expressed as

$$
I_{\text {effective }}=I_{\text {leg }}+I_{\text {spine }}
$$

where $I_{l e g}$ is equal to $b_{\text {leg }} h_{\text {leg }}^{3} / 12$ and $I_{\text {spine }}$ is expressed as

$$
I_{\text {spine }}=\frac{E_{\text {spine }} b_{\text {spine }} h_{\text {spine }}^{3}}{12 E_{\text {leg }}}
$$

This formulation is an adaptation of the one presented in [51]. The ratio of $E_{\text {spine }}$ to $E_{\text {leg }}$ is a common expression used to account for situations in which members subject to bending are made of more than one material.

Since the PRB model assumes a uniform cross-section, the model cannot be used to estimate the leg stiffness range and tip trajectory at intermediate stiffness settings. The finite element method can be used to produce the needed information; however, this requires a larger investment of time. To expedite the design process, we have determined that a stiffness setting near the angular position of $50^{\circ}$ (see Fig. 9B) leads to the greatest tip trajectory deviation. Therefore if one can design the tip trajectory at this setting to approximately match the behavior at the stiffness extremes, then the intermediate settings should also closely approximate the same behavior.

\subsection{Experimental Leg Characterization}

To observe the leg deflection behavior and to validate the PRB model, the leg testing experimental apparatus presented in Section IIIA as shown in Fig. 13 was modified to measure an applied load and to record the resulting deflection path. The Micos linear stage and an AMTI HE6x6 force plate were rigidly connected to an aluminum base plate. The C-leg's aluminum hip was anchored to the linear stage platform and the C-leg was cantilevered out from the platform. An aluminum clamp was affixed to the leg at the position indicated by Marker 2 in Fig. 13A. One end of a flexible steel

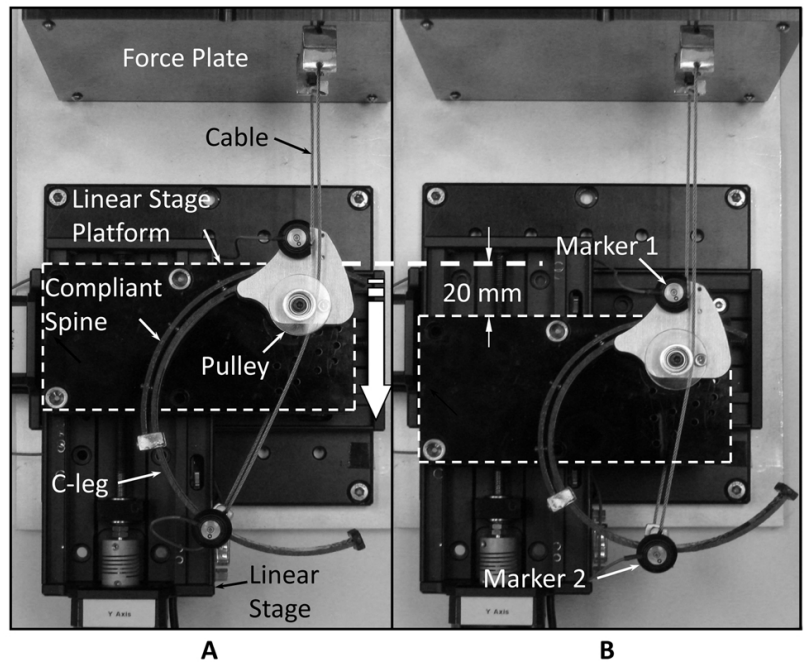

Fig. 13. Top view of experimental set-up. A) Linear Stage is in the home position and leg is undeflected. B) Platform has been moved a distance, $\mathrm{d}$, and the leg is deflected.

cable was anchored to the force plate while the other was connected to the leg clamp. A pulley was anchored to the hip to provide a rolling contact point and to make the cable normal to the the force plate's surface. The linear stage was commanded to translate (see Fig. 13B) the hip a distance of $20 \mathrm{~mm}$ at $10 \mathrm{~mm} / \mathrm{s}$ in the $\mathrm{y}$-direction (given by large downward pointing grey arrow on the right side of Fig. 13A). The force plate collected the reaction forces at the loading point (Marker 2) at a sampling rate of $200 \mathrm{~Hz}$. An Optotrak 3020 motion capture system was used to capture the position of Markers 1 and 2 also at a sampling rate of $200 \mathrm{~Hz}$. This was repeated for each of the leg stiffness settings $0-4$ by shifting the compliant spine (see Fig. 9B) along the length of the Cleg. The 6-ply fiberglass C-leg and spine were constructed with an alternating $50 / 50$ blend ratio where $50 \%$ of the plies where angled at $45^{\circ}$ while the other half were angled at $0^{\circ}$. The leg inner diameter is $114 \mathrm{~mm}$ with a thickness of 2.25 $\mathrm{mm}$ and a width of $18 \mathrm{~mm}$. We estimate the Young's modulus value to be $9.65 \mathrm{GPa}$.

\subsubsection{Stiffness Results}

In Fig. 14 the experimental results of the load measured against the deflection in the radial direction demonstrate that the stiffness increases monotonically. The stiffness, which is indicated as a slope value, $\mathrm{k}$, next to each curve, doubled between the two stiffness extremes. This was expected as the only difference between the two extremes was a doubling of the moment of inertia. Its also worth noting that the stiffness increase from leg stiffness setting (LSS) 0 and LSS 1 is approximately $9 \%$ for this configuration. In future models, LSS1 could be the home position to allow the leg to reach higher stiffness settings faster without significantly limiting the stiffness range. 


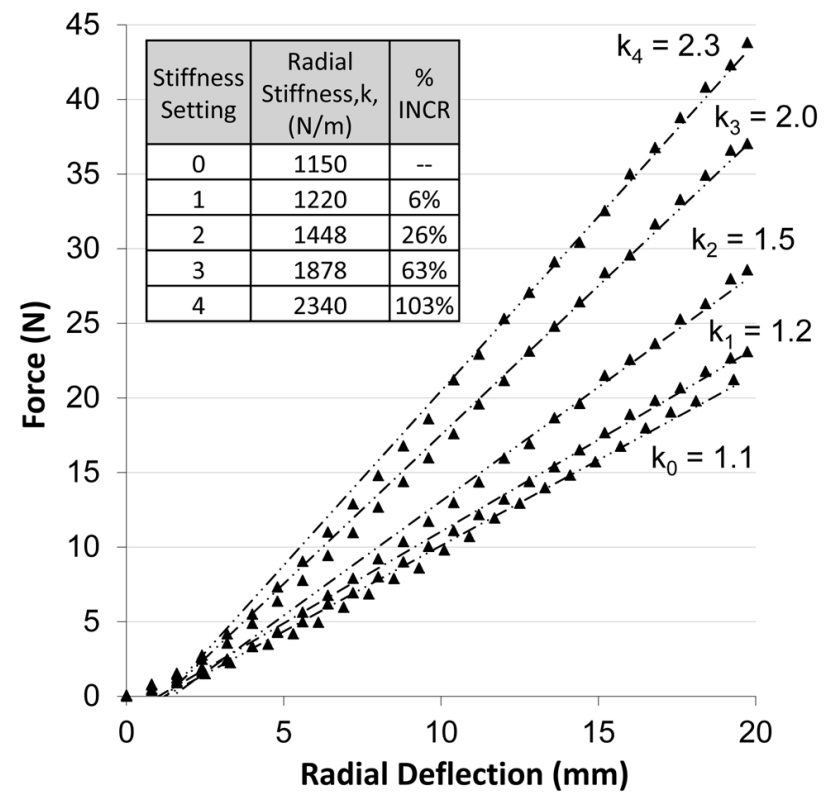

Fig. 14. Spring force response at four different leg stiffness settings each with a curve fit (dotted line) applied to the loading phase.

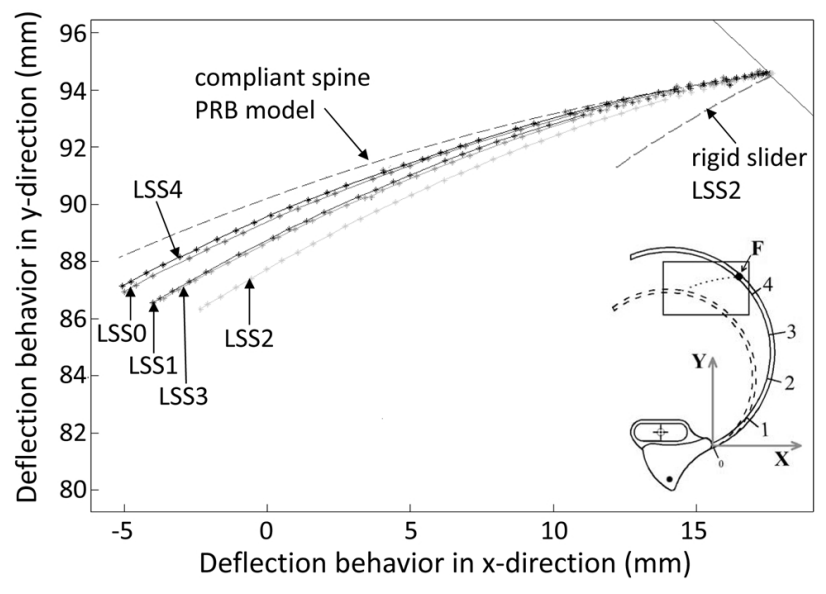

Fig. 15. Deflection path of leg for various stiffness settings.

\subsubsection{Deflection Orientation Results}

In Fig. 15 the actual xy-deflection of the leg under load is presented. The bottom right image in Fig. 15 provides a bearing for the location and orientation of the xy-axis while the rectangle reflects the results window. For the range of the stiffness settings tested the deflection paths showed low variability. In particular, the deflection path of the two extreme stiffnesses (i.e. LSS0 and LSS4) were almost identical and varied by no more than $0.5 \mathrm{~mm}$ from each other. At maximum deflection, these results were also within $1 \mathrm{~mm}$ of the deflection path predicted by the compliant spine PRB model, which for a total deflection of $20 \mathrm{~mm}$ in the y-direction, represents about a 5\% estimation error. As expected, the deflection path at LSS2 showed the most deviation. At maximum deflection, the y-component deviation was approximately 2 $\mathrm{mm}$, which represents roughly a $10 \%$ difference from the

\begin{tabular}{|c|c|c|c|c|c|}
\hline \multicolumn{7}{|c|}{ Fixed Stiffness C-leg } \\
\hline Layers of Fiberglass & $5 \mathrm{~L}$ & $6 \mathrm{~L}$ & $6.5 \mathrm{~L}$ & $7 \mathrm{~L}$ & $9 \mathrm{~L}$ \\
\hline $\begin{array}{c}\text { Relative Leg Stiffness } \\
\text { (RLS) }\end{array}$ & 0.85 & 1 & 1.3 & 1.6 & 3.1 \\
\hline $\begin{array}{c}\text { Radial Leg Stiffness } \\
(\mathrm{N} / \mathrm{m})\end{array}$ & 1090 & 1280 & 1660 & 2050 & 3970 \\
\hline
\end{tabular}

Table 3. This table specifies the conversion from relative leg stiffness to radial leg stiffness for the variety of fixed stiffness C-legs.

compliant spine PRB curve. For comparison purposes, the same tangential force that produced the deflection path for LSS2 was applied to a rigid slider PRB model also at LSS2. The rigid slider tuning method clearly produces very different spring behavior (see curve labled 'Rigid Slider LSS2'). The stiffness is much larger given by the short deflection path, and the characteristic radius is much shorter creating a steep deflection path. It should be noted that achieving consistent deflection behavior for all stiffnesses while achieving a large deflection range are two competing objectives. If the compliant spine is too soft then the deflection path will be consistent; however, the stiffness range will be very small. Similarly, if the compliant spine is too stiff, the deflection path and stiffness range will begin to reflect the rigid slider model. Therefore while some deviation in deflection behavior is expected, through proper material selection and geometries this can be minimized while still achieving a considerable stiffness range. As these tests indicate, these new compliant-slider fiberglass legs overcome the shortcomings identified for the earlier rigid slider design.

\section{Dynamic Locomotion Testing}

\subsection{Experimental Procedure}

Previous optimization studies on RHex primarily focused on boosting robot performance through gait parameter adjustment [52]. One constant stiffness C-leg was used and no other leg stiffnesses were explored. While these efforts were productive, the question remains concerning the effect of varying leg compliance for a RHex-style robot. In particular, what are the consequences of overly soft or stiff legs on running performance.

In the following preliminary optimization experiments, we sought to understand the role of leg compliance in EduBot. We initially explore this topic with constant stiffness C-legs so as to eliminate any unwanted effects a tunable leg might introduce. Five sets of C-legs were constructed from S2-6781 pre-preg fiberglass. The stiffness of each set was varied during manufacturing by either changing the number of layers of fiberglass or the leg width (see Table 3 for resulting leg stiffnesses). The softest leg used 5 layers (5L) while the stiffest leg used 9 layers $(9 \mathrm{~L})$. The $9 \mathrm{~L}$ is approximately $3.6 \mathrm{x}$ stiffer than the $5 \mathrm{~L}$ leg. The leg labeled $6.5 \mathrm{~L}$ is actually a 7 layer $(7 \mathrm{~L})$ leg with a width that was reduced from $18 \mathrm{~mm}$ to $15 \mathrm{~mm}$. This was done in order to quickly achieve a leg stiffness that fell in between a $6 \mathrm{~L}$ and a $7 \mathrm{~L}$.

To identify suitable gaits for each leg stiffness, an auto- 
mated Nelder-Mead optimization routine was used similar to the method implemented on RHex [52]. A Vicon motion capture system was used to control the robot during all aspects of the experiment. Reflective tracking markers mounted to the robot shell allowed the controller to accurately and repeatably steer the robot from one end of the test arena, know as an end zone, to the other. The length of each run measured approximately 18 feet with the first $35 \%$ reserved for acceleration, and the last $5 \%$ reserved for deceleration. During each trial (i.e. running from one end zone to the other), the average power and average velocity were recorded. These values were then used to calculate cost function. For this we used average specific resistance, SR, which is a non-dimensionalized parameter that characterizes energy efficiency as the ratio of average power in over average power out

$$
S R=\frac{P_{a v g}}{m g v_{a v g}}
$$

where $\mathrm{P}_{a v g}$ is the average power consumed, $\mathrm{m}$ is the mass of the robot $(3.3 \mathrm{~kg})$ and any payloads, $g$ is gravity, and $\mathrm{v}_{\text {avg }}$ is the average forward speed. It should be noted that for the purposes of experimentation, we assume that lower $S R$ values are the signature of relatively stable gaits while higher $S R$ values are the signature of an unstable and energetically wasteful gaits. For each leg stiffness one and in many cases two Nelder-Mead descents were performed for each combination of four different payloads including $0 \mathrm{~kg}$, $0.45 \mathrm{~kg}, 0.91 \mathrm{~kg}$, and $1.36 \mathrm{~kg}$. The payloads were in the form of steel plates that were secured to the belly of the robot and positioned so as not to shift the robot's projected center of mass. The robot generally converged to suitable gaits after $90+$ trials

\subsection{Experimental Results}

For the tested range of leg stiffness, it can be observed in Figs. 16 and 17 that softer legs posted better speed and efficiency results. In fact Fig. 17 shows that the no-load forward speed for a $9 \mathrm{~L}, 7 \mathrm{~L}, 6.5 \mathrm{~L}, 6 \mathrm{~L}$, and $5 \mathrm{~L}$ leg is approximately $0.85,1.35,1.5,2.1$, and $2 \mathrm{~m} / \mathrm{s}$ respectively (though under the right conditions we were able to attain forward velocities of $2.6 \mathrm{~m} / \mathrm{s}$ with the $6 \mathrm{~L} \mathrm{leg}$ ). This outcome contradicted some of our earlier held expectations derived from our experience with RHex. In a body mass adjusted comparison between RHex and EduBot leg stiffnesses in [53], it was estimated that $6 \mathrm{~L}$ EduBot legs are 3.5 times more compliant than the RHex legs used for gait optimization studies in [52]. It should be highlighted that RHex achieved some of its fastest optimized speeds in this study. With EduBot we observe instability in the higher leg stiffness regime where as lower leg stiffnesses maintained robot stability in the face of uneven tripod touchdown. Notwithstanding the extensive mathematical analysis of the SLIP template [54] and its clockdriven excitation in the RHex anchor [55] there is still insufficient theoretical understanding of the interplay between the clock, controller, mechanical parameters in stabilizing RHex gaits that this observed behavior is probably best explained through an example. If a stiff leg touches down early (i.e. closer to the hip than to the toe) then the leg is essentially a rigid element inelastically colliding with the ground. Consequently, the leg falls behind its desired position according to the Beuhler Clock for which the PD controller tries to minimize by inserting considerable torque in a short time interval. This imparts pitching and rolling moments to the robot body that cause imbalance on the next tripod touchdown. Therefore, with this particular controller, stiff legs appear to narrow the region of stable gaits. Soft legs, on the other hand, are more capable of deflecting and absorbing energy even if the leg touches down early. This significantly reduces the severity of ground reaction forces that contribute to stance phase imbalance.

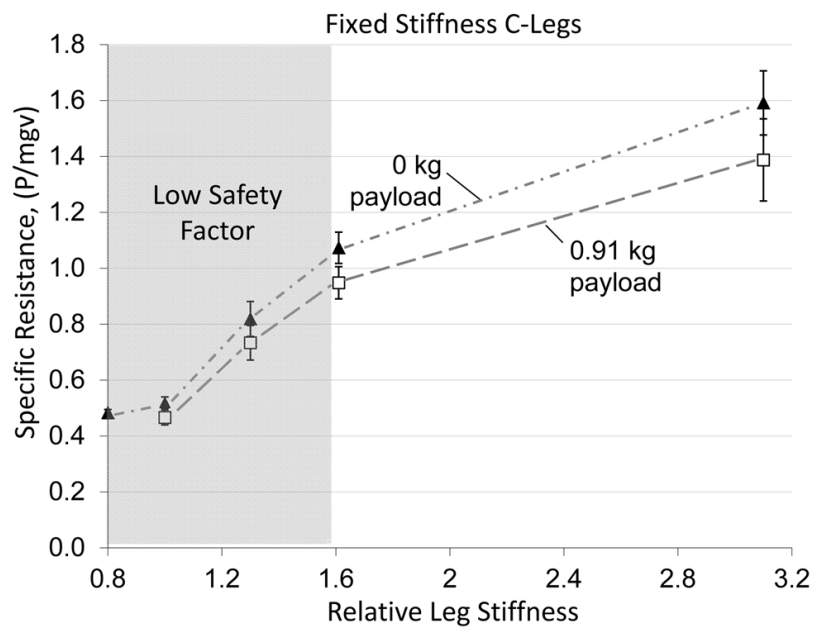

Fig. 16. Preliminary experimental results showing specific resistance against relative leg stiffness.

While the 5L and 6L legs ran most efficiently for the range of payloads, there was an increased occurrence of leg failure as the payload increased. In fact, 5L experiments were terminated after adding $0.45 \mathrm{~kg}$ because three legs broke in one optimization. This is one of the drawbacks of the dual nature of passive compliant legs (i.e. as a structural support appendage and a spring). In a typical Nelder-Mead decent, the legs are subjected to gaits that create uneven leg loading events that place considerable stress on individual legs. We believe these conditions are indicative of the abuse the legs may experience while running on rough terrain. Therefore, if a leg can not survive a simple optimization then it certainly is not suitable for real world conditions.

Without any modifications to the C-leg design there appears to be a trade-off between leg survivability and speed and efficiency. To illustrate this concept of survivability, a shaded region labeled Low Safety Factor has been incorporated in Fig. 16, which is based on recorded leg failures during the optimizations. We claim that any robot activity in 


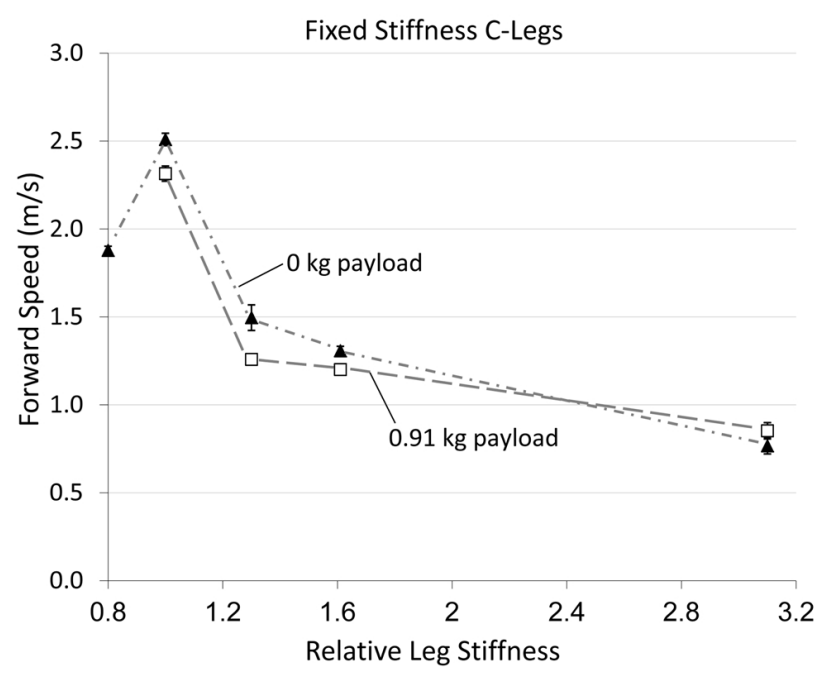

Fig. 17. Preliminary experimental results comparing relative leg stiffness against top forward speed.

the shaded zone has a higher probability of leg failure (especially with larger payloads and/or on uneven terrain) while operations outside this region are less likely to exceed the leg's material limits. It should be recognized that the width of this region would vary depending on the payload, speed, and terrain.

\subsection{Resulting Design Modifications}

There are two significant points one can draw from these results. The first relates to the desirable stiffness range. The robot appears to run faster and more efficiently with leg stiffnesses less than or equal to a $6 \mathrm{~L} \mathrm{leg}$. This suggests that to uncover the value of tunable stiffness legs for this robotic platform the stiffness range must operate in this realm or lower. This exposes the limitation of the C-leg design and brings us to the second point: a modification to the C-leg design is necessary to overcome this limitation. If the baseline stiffness is designed to survive most loading scenarios then as the experimental results demonstrate, faster and more efficient gaits are simply out of reach. Therefore to safely operate in a lower stiffness realm $(\mathrm{k}<1250 \mathrm{~N} / \mathrm{m})$, we propose a simple solution in the form of a mechanical stop whereby lower stiffness legs are prevented from deflecting past their material limit. Based on these findings a final leg design with a stiffness range from 640 to $1280 \mathrm{~N} / \mathrm{m}$ was built with a second, smaller 'C-shaped' structure that serves as a safety, or mechanical stop, for extreme loading conditions. The final design is shown in Fig. 18.

\section{Conclusions and Future Work}

We have developed a robust, self-locking, structurecontrolled, tunable stiffness leg for implementation on a dynamic hexapedal robot. We have shown that with a proper selection of materials and geometries, the proposed tunable leg can achieve a factor of two or more change in stiffness

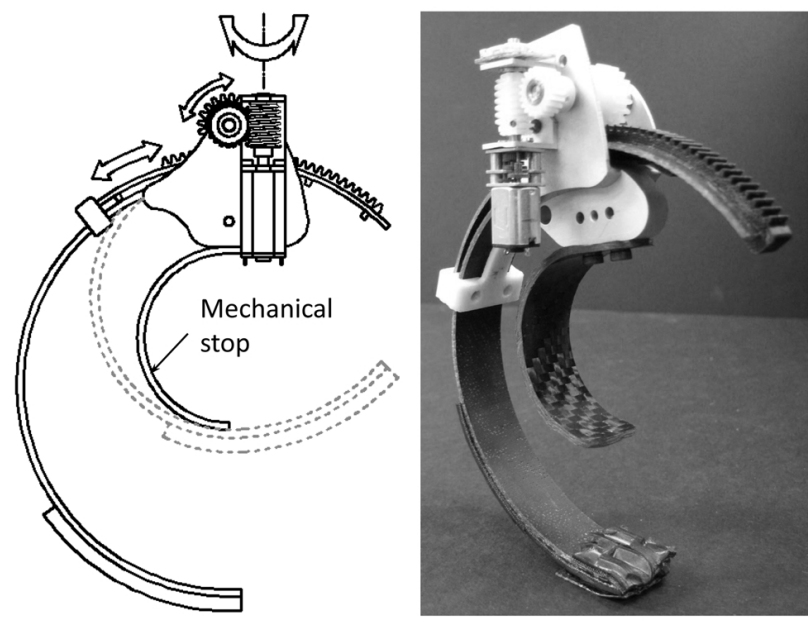

Fig. 18. Final leg design: left) illustration of mechanical stop, right) photo of final assembly.

without a significant change in deflection behavior. Several materials have been considered; however, we have found that composite materials offer the best combination of energy storage capacity, high yield strength, ease of manufacturing, and Young's modulus control.

We have also shown experimental evidence that varying the stiffness of robot legs can improve the locomotion performance both in terms of speed and efficiency. As a point of comparison, the best documented RHex gait achieved a forward speed of $2.7 \mathrm{~m} / \mathrm{s}$ with a specific resistance of 0.84 [52]. When EduBot's gait was optimized for a range of leg stiffnesses, we converged to a forward speed of $2.5 \mathrm{~m} / \mathrm{s}$ (and in some cases $2.6 \mathrm{~m} / \mathrm{s}$ ) with a specific resistance of 0.5 , which makes EduBot locomotion roughly $40 \%$ more efficient than RHex at nearly the same speed and 1/3 the weight.

With a proper communication and control strategy these integrated 'smart' legs will be capable of run-time adaptations to changing environmental conditions, moving us one step closer to truly agile dynamic robots. As part of our ongoing work, we will explore through experimentation on EduBot the benefits and costs of mechanically tuning leg compliance. It is our intention that these new tunable legs will be used on our robotic platform to experimentally validate simulation results and hypotheses about the effect of variable stiffness legs on the stability and efficiency of legged locomotion.

\section{Acknowledgments}

This work was partially supported by the NSF FIBR grant \#0425878 and the IC Postdoctoral Fellow Program under grant number HM158204-1-2030. Some of the component pieces of the present account have been offered in preliminary form in various conferences including [56] (rigid slider design and analysis), [57] (compliant slider design and analysis), and [58] (empirical optimization studies). 


\section{References}

[1] Dickinson, M., Farley, C., Full, R., Koehl, M., Kram, R., and Lehman, S., 2000. "How animals move: an integrative view.". Science, 288(5463), pp. 100-6.

[2] Cavagna, G. A., Heglund, N. C., and R., T. C., 1977. "Mechanical work in terrestrial locomotion: Two basic mechanisms for minimizing energy expenditure". American Journal of Physiology, 233.

[3] Blickhan, R., 1989. "The spring-mass model for running and hopping". J. Biomech., 22(11-12), pp. 12171227.

[4] Blickhan, R., and Full, R. J., 1993. "Similarity in multilegged locomotion: Bounding like a monopod". Journal of Comparative Physiology, 173(5), pp. 509-517.

[5] Raibert, M. H., 1986. Legged robots that balance. MIT Press series in artificial intelligence. MIT Press, Cambridge, Mass.

[6] Buehler, M., Battaglia, R., Cocosoc, A., Hawker, G., Sarkis, J., and Yamazaki, K., 1998. "Scout: A simple quadruped that walks, climbs, and runs". International Journal of Robotics Research, 13(2), pp. 1707-1712.

[7] Papdopoulos, D., and Buehler, M., 2000. "Stable running in a quadruped robot with compliant legs". In Proc. of the IEEE International Conference on Robotics and Automation, pp. 444-449.

[8] Smith, J. A., and Poulakakis, I., 2004. "Rotary gallop in the untethered quadrupedal robot scout ii". In Proc. of the IEEE/RSJ International Conference on Intelligent Robots and Systems, Vol. 3, pp. 2556-2561.

[9] Saranli, U., Buehler, M., and Koditschek, D. E., 2000. "Design, modeling and preliminary control of a compliant hexapod robot". In Proceedings - IEEE International Conference on Robotics and Automation, Vol. 3, pp. 2589-2596.

[10] Cham, J. G., Karpick, J., Clark, J. E., and Cutkosky, M. R., 2001. "Stride period adaptation for a biomimetic running hexapod". In International Symposium of Robotics Research.

[11] Ferris, D., Louie, M., and Farley, C., 1998. "Running in the real world: Adjusting leg stiffness for different surfaces". In Proceedings of the Royal Society London, Vol. 265, pp. 989-993.

[12] Brown, I., and Loeb, G., 2000. "A reductionist approach to creating and using neuromusculoskeletal models". In Biomechanics and Neural Control of Posture and Movement, J. Winters and P. Crago, eds. Springer-Verlag, pp. 148-163.

[13] Holmes, P., Full, R., Koditschek, D., and Guckenheimer, J., 2006. "The dynamics of legged locomotion: Models, analyses, and challenges". SIAM Review, 48(2), pp. 207-304.

[14] Jindrich, D., and Full, R., 2002. "Dynamic stabilization of rapid hexapedal locomotion". Journal of Experimental Biology, 205(18), pp. 2803-2823.

[15] Van Ham, R., Van Damme, M., Vanderborght, B., Verrelst, B., and Lefeber, D., 2006. "Maccepa: The mechanically adjustable compliance and controllable equilibrium position actuator". In Proceedings of
CLAWAR, pp. 196-203.

[16] Daerden, F., and Lefeber, D., 2001. "The concept and design of pleated pneumatic artificial muscles". International Journal of Fluid Power, 2(3), pp. 41-50.

[17] Hurst, W., Chestnutt, J., E., and Rizzi, A., A., 2004. "An actuator with physically variable stiffness for highly dynamic legged locomotion". In Proceedings - IEEE International Conference on Robotics and Automation, pp. 4662-4667.

[18] Hurst, J., 2008. "The role and implementation of compliance in legged locomotion". $\mathrm{PhD}$ thesis, Carnegie Mellon University.

[19] Alexander, R. M., 1990. "Three uses for springs in legged locomotion". International Journal of Robotics Research, 9(2), pp. 53-61.

[20] Komsuoglu, H., 2007. Towards a comprehensive infrastructure for construction of modular and extensible robotic systems. Tech. rep., Dept. of Computer and Information Science, University of Pennsylvania.

[21] Weingarten, J., Koditschek, D., Komsuoglu, H., and Massey, C., 2007. "Robotics as the delivery vehicle: A contextualized, social, self paced, engineering education for life-long learners". In RSS: Robot Science and Systems, p. 314.

[22] Pratt, G., and Williamson, M., 1995. "Series elastic actuators". Intelligent Robots and Systems, IEEE/RSJ International Conference on, 1, p. 399.

[23] Fukuoka, Y., Kimuar, H., and Cohen, A. H., 2003. "Adaptive dynamics walking of a quadruped robot on irregular terrain based on biological concepts". International Journal of Robotics Research, 22(3-4), pp. 187202.

[24] Nichol, J. G., Palmer, L., Singh, S., Orin, D., and Waldron, K., 2004. "System design of a quadrupedal galloping machine". International Journal of Robotics Research, 23, pp. 1013-1027.

[25] Lambrecht, B. G. A., Horchler, A. D., and Quinn, R. D., 2005. "A small, insect-inspired robot that runs and jumps". Proc. of the IEEE International Confer-ence on Robotics and Automation, pp. 1252-1257.

[26] Quinn, R. D., Nelson, G. M., Bachmann, R. J., Kingsley, D. A., Offi, J., and Ritzmann, R. E., 2001. "Insect designs for improved robot mobility". In Proceedings of CLAWAR 2001, Karlsruhe, Germany, D. Berns, ed., pp. 69-76.

[27] Cham, J. G., 2002. "On stability and performance in open-loop running". $\mathrm{PhD}$ thesis, Stanford University.

[28] Saranli, U., Buehler, M., and Koditschek, D. E., 2001. "Rhex: A simple and highly mobile hexapod robot". International Journal of Robotics Research, 20(7), pp. 616-631.

[29] Altendorfer, R., Moore, N., Komsuoglu, H., Buehler, M., Brown H. B, J., McMordie, D., Saranli, U., Full, R., and Koditschek, D. E., 2001. "Rhex: A biologically inspired hexapod runner". Autonomous Robots, 11(3), pp. 207-213.

[30] Lin, P.-C., Komsuoglu, H., and Koditschek, D., 2005. "Sensor data fusion for body state estimation in a hexa- 
pod robot with dynamical gaits". In ICRA, pp. 47334738.

[31] Lopes, G., and Koditschek, D., 2007. "Visual servoing for nonholonomically constrained three degree of freedom kinematic systems". I. J. Robotic Res., pp. 715736.

[32] Clark, J. E., 2004. "Design, simulation, and stability of a hexapedal running robot". PhD thesis, Stanford University.

[33] Burden, S., Clark, J. E., Weingarten, J .and Komsouglu, H., and Koditschek, D. E., 2007. "Heterogeneous leg stiffness and roll in dynamic running". In IEEE - International Conference of Robotics and Automation.

[34] Moore, E. Z., 2002. "Leg design and stair climbing control for the RHex robotic hexapod". Master's thesis, McGill University, Jan.

[35] Vanderborght, B., Van Ham, R., Lefeber, D., Sugar, T., and Hollander, K., 2009. "Comparison of mechanical design and energy consumption of adaptable, passivecompliant actuators". International Journal of Robotics Research, 28(1), pp. 90-103.

[36] Beyl, P., Vanderborght, B., Van Ham, R., Van Damme, M., Versluys, R., and Lefeber, D., 2006. "Compliant actuation in new robotic applications". In NCTAM06 7th National Congress on Theoretical and Applied Mechanics.

[37] Morita, T., and Sugano, S., 1995. "Design and development of a new robotic joint using a mechanical impedance adjuster". In IEEE International Conference on Robotics and Automation, Vol. 3, pp. 2469-2475.

[38] Hollander, K., Sugar, T., and Herring, D., 2005. "Adjustable robotic tendon using a 'jack spring'tm". In IEEE International Conference on Rehabilitation Robotics.

[39] Tabata, O., Konishi, S., Cusin, P., Ito, Y., Kawai, F., Hirai, S., and Kawamura, S., 1999. "Microfabricated tunable stiffness device". Proc. of the 13th Annual Int. Conf. On Micro Electro Mechanical Systems.

[40] Moore, E. Z., Campbell, D., Grimminger, F., and Buehler, M., 2002. "Reliable stair climbing in the simple hexapod 'rhex"'. In Proceedings - IEEE International Conference on Robotics and Automation, Vol. 3, pp. 2222-2227.

[41] Lin, P.-C., 2005. "Proprioceptive sensing for a legged robot". PhD thesis, University of Michigan.

[42] Lin, P.-C., Komsuoglu, H., and Koditschek, D. E., 2005. "A leg configuration measurement system for full body posture estimates in a hexapod robot". IEEE Transactions on Robotics, 21(3), pp. 411-422.

[43] Howell, L. L., 2001. Compliant Mechanisms. Wiley, New York.

[44] Howell, L. L., and Midha, A., 1996. "Parametric deflection approximations for initially curved, largedeflection beams in compliant mechanisms". In Proceedings of the ASME Design Engineering Technical Conference.

[45] Merz, R., B., P. F., Ramaswami, K., Terk, M., and Weiss, L. E., 1994. "Shape deposition manufacturing".
In Proceedings of the Solid Freeform Fabrication Symposium.

[46] Cham, J. G., Bailey, S. A., and Cutkosky, M. R., 2000. "Robust dynamic locomotion through feedforwardpreflex interaction". In ASME IMECE Proceedings.

[47] Chitta, S., Karabas, M., Galloway, K., and Kumar, V., 2006. "Robotrikke: Design, modeling and experimentation with a robotic trikke". In Proceedings of the ASME Design Engineering Technical Conference.

[48] Dollar, A., and Howe, R., 2005. "Design and evaluation of a robust compliant grasper using shape deposition manufacturing". In Proceedings of the ASME Design Engineering Technical Conference.

[49] Shigley, J., 1977. Mechanical Engineering Design. McGraw-Hill, New York.

[50] Barbero, E., 1999. Introduction to Composite Materials Design. Taylor and Francis Inc.

[51] Kawamura, S., Yamamoto, T., Ishida, D., Ogata, T., Nakayama, Y., Tabata, O., and Sugiyama, S., 2002. "Development of passive elements with variable mechanical impedance for wearable robots". In IEEE Interternational Conference on Robotics and Automation, pp. 248-253.

[52] Weingarten, J., Groff, R., and Koditschek, D., 2004. "A framework for the coordination of legged robot gaits". In IEEE International Conference of Robotics, Automation and Mechatronics, Singapore.

[53] Galloway, K., 2010. "Passive variable compliance for dynamic legged robots". $\mathrm{PhD}$ thesis, University of Pennsylvania.

[54] Ghigliazza, R. M., Altendorfer, R., Holmes, P., and Koditschek, D., 2005. "A simply stabilized running model”. SIAM Rev., 47, March, pp. 519-549.

[55] Altendorfer, R., Koditschek, D., and Holmes, P., 2004. "Stability analysis of a clock-driven rigid-body slip model for rhex". I. J. Robotic Res., 23(10-11), pp. 1001-1012.

[56] Galloway, K., Clark, J., and Koditschek, D., 2007. “Design of a multi-directional variable stiffness leg for dynamic runnings". In ASME Int. Mech. Eng. Congress and Exposition.

[57] Galloway, K. C., Clark, J. E., and Koditschek, D. E., 2009. "Design of a tunable stiffness composite leg for dynamic locomotion". ASME Conference Proceedings, 2009(49040), pp. 215-222.

[58] Galloway, K., Clark, J., Yim, M., and Koditschek, D., 2011. "Experimental investigations into the role of passive variable compliant legs for dynamic robotic locomotion”. In Robotics and Automation (ICRA), 2011 IEEE International Conference on, pp. 1243 -1249. 